\title{
Vinyl Ester Oligomer Crosslinked Porous Polymers Prepared via Surfactant-Free High Internal Phase Emulsions
}

\author{
Yun Zhu, Ye Hua, Shengmiao Zhang, Jianding Chen, and Chun Pu Hu \\ Shanghai Key Laboratory of Advanced Polymeric Materials, Key Laboratory for Ultrafine Materials of Ministry of Education, \\ School of Materials Science and Engineering, East China University of Science and Technology, Shanghai 200237, China
}

Correspondence should be addressed to Shengmiao Zhang, shmzhang@ecust.edu.cn

Received 20 February 2012; Accepted 12 March 2012

Academic Editor: Sevan P. Davtyan

Copyright ( 2012 Yun Zhu et al. This is an open access article distributed under the Creative Commons Attribution License, which permits unrestricted use, distribution, and reproduction in any medium, provided the original work is properly cited.

Using vinyl ester resin (VER) containing styrene (or methyl methacrylate) and vinyl ester oligomer (VEO) as external phase, Pickering high internal phase emulsions (Pickering HIPEs) having internal phase volume fraction of up to 95 vol\% were prepared with copolymer particles as sole stabilizer. Polymerizing the external phase of these Pickering HIPEs led to porous polymers (polyPickering-HIPEs). Compared to the polystyrene- (PS-) based poly-Pickering-HIPEs which were prepared with mixture of styrene and divinylbenzene (DVB) as crosslinker, the poly-Pickering-HIPEs herein showed much higher elastic modulus and toughness. The elastic modulus of these poly-Pickering-HIPEs increased with increasing the VEO concentration in the external phase, while it decreased with increasing internal phase volume fraction. Increasing VEO concentration in the external phase also resulted in a decrease in the average void diameter as well as a narrow void diameter distribution of the resulting poly-Pickering-HIPEs. In addition, there were many small pores in the voids surface caused by the volume contraction of VER during the polymerization, which suggests a new method to fabricate porous polymers having a well-defined hierarchical pore structure.

\section{Introduction}

High internal phase emulsions (HIPEs) are defined as emulsions, in which the internal phase occupies higher than $74.05 \mathrm{vol} \%$ of the emulsion. These emulsions are important for a wide range of applications in the food, cosmetic, pharmaceutical, and petroleum industries $[1,2]$. Recently, increasing interests are attracted to use the emulsions as templates for the production of highly porous polymers (known as polyHIPEs) [3-16]. Such materials are prepared by polymerizing the thin films that surround the droplets of the HIPEs if the continuous phase contains one or more monomeric species that are polymerizable. Conversional HIPEs are commonly stabilized against coalescence by large fractions (5-50 vol\%) of nonionic surfactant [2, 13]. The sheer quantity of surfactant required to stabilize HIPEs both limits properties and is a major cost factor.

To eliminate the surfactants used in HIPEs, the authors, and other groups, have prepared HIPEs using organic or inorganic particles as stabilizer [3-9, 16]. Such emulsions are known as Pickering HIPEs. And the porous polymers based on this type of emulsions are known as poly-PickeringHIPEs. By far, the most widely studied monomer used in poly-Pickering-HIPEs is styrene (St). Usually, a varied amount of hydrophobic crosslinker, such as divinylbenzene (DVB), is also added to enhance the structural stability $[16,17]$. This porous polymer is being considered for many applications such as filtration media and support for heterogenic catalytic reactions and has even been explored for tissue engineering application, that is, as scaffold to support the growth of osteoblast cells [17-20]. However, it is difficult to use polystyrene- (PS-) based poly-PickeringHIPEs as commercial production because of their undesirable properties, such as brittleness and chalkiness.

In order to enhance the mechanical properties of PSbased poly-Pickering-HIPEs, during the course of the work on developing novel poly-Pickering-HIPEs, the authors undertook the preparation of species containing vinyl ester resin (VER). Generally, VER is a mixture of St with methacrylated epoxy compounds (often referred to as vinyl ester oligomer (VEO)) $[21,22]$. In this case, styrene is a reactive diluent, and $\mathrm{VEO}$ serves as a crosslinking agent for 


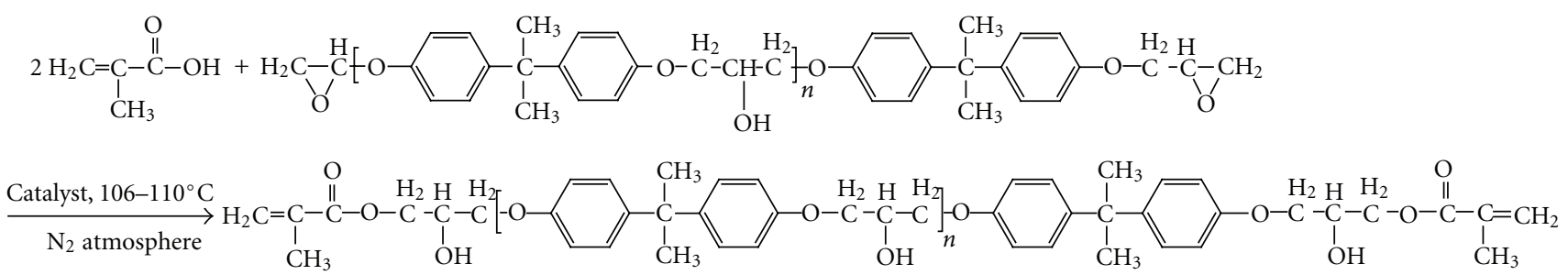

FIgURE 1: Synthetic schemes of VEO.

preparing poly-Pickering-HIPEs. VER has superior properties relative to unsaturated polyester systems and is easy to process.

In this study, VER-based poly-Pickering-HIPEs were prepared using poly(styrene-co-methyl methacrylate-co-acrylic acid) (P(St-co-MMA-co-AA ) copolymer particles as stabilizer. The elastic modulus of the porous polymers was investigated, and the morphology of these porous materials was also tailored by changing internal phase (aqueous phase) volume fraction and VEO concentration in organic phase. In addition, poly(methyl methacrylate)- (PMMA-) based polyPickering-HIPEs were also prepared with mixture of methyl methacrylate (MMA) and VEO as organic phase.

\section{Experimental Section}

2.1. Materials. Styrene (St, 99\%), methyl methacrylate (MMA, 99\%), and acrylic acid (AA, 98\%) were purchased from Shanghai Lingfeng Chemical Reagent Ltd. Co. and were distilled under a reduced-pressure nitrogen atmosphere before use. Divinylbenzene (DVB, 80\%, the remainder being m- and p-ethylstyrene,) was purchased from Sigma Aldrich and was purified by passing through neutral chromatographic aluminum oxide to eliminate the inhibitor. Ammonium persulphate (APS, 98\%) was purchased from Shanghai Lingfeng Chemical Reagent Ltd. Co. and was purified via recrystallization. Sodium chloride $(\mathrm{NaCl})$ was purchased from Shanghai Experiment Reagent Ltd. Co. and was used as received. E-51 epoxy resin (bisphenol A-type, 0.51 epoxide equivalent/100 g resin) was produced by Shanghai Synthetic Resin. $\alpha$-methacrylic acid ( $\alpha$-MAA, 97\%), tetrabutyl ammonium bromide (TBAB, 99\%), and 1,4-hydroquinone (HQ, 99\%), were purchased from Shanghai Lingfeng Chemical Reagent Ltd. Co. and were used as received. Water was fresh deionized.

2.2. Synthesis of Vinyl Ester Oligomer. VEO was synthesized by the reaction of epoxy resin and methacrylic acid as shown in Figure 1. All the synthesis and characterization of VEO were described in detail elsewhere [22-24]. Thus, a mixture of $1 \mathrm{~mol} \mathrm{E}-51, \mathrm{TBAB}(0.1 \mathrm{wt} \%$, relative to E-51), and HQ (0.03 wt \%, relative to E-51) was added to a $500 \mathrm{~mL}$ threeneck flask equipped with an overhead leaf-shaped stirrer paddle and a condenser. After the nitrogen was bubbled through the mixture for $30 \mathrm{~min}$, the temperature of the mixture was elevated to $90^{\circ} \mathrm{C}$, and then $2 \operatorname{mol} \alpha$-MAA was added dropwise to the mixture with stirring. After the addition of $\alpha$ MAA, the temperature of the mixture was elevated gradually to $110^{\circ} \mathrm{C}$ and kept at $110^{\circ} \mathrm{C}$. When the acid number (AN) of the mixture was below $6 \mathrm{mgKOH} / \mathrm{g}$ (determined according to $\mathrm{GB} 2895-82$ ), the reaction continued under vacuum to reduce the $\mathrm{AN}$ further $(\mathrm{AN}<0.6 \mathrm{mgKOH} / \mathrm{g})$, and the $\mathrm{VEO}$ was obtained. The St (or MMA) was then introduced into this VEO to prepare the VER (VEO/St (or MMA) $=64 / 36$, wt/wt). The resulting VER was designated as VER-St (or VER-MMA).

2.3. Preparation and Characterization of $P(S t-c o-M M A-c o-$ AA) Copolymer Particles. The preparation of monodisperse $\mathrm{P}$ (St-co-MMA-co-AA) particles has been reported elsewhere [25]. Water $(100 \mathrm{~mL})$ containing APS of $7.0 \times 10^{-3} \mathrm{~mol} \mathrm{~L}^{-1}$ was added to a four-necked glass reactor $(250 \mathrm{~mL})$ with an overhead leaf-shaped stirrer paddle and a condenser. Then the mixture consisted of $18.2 \mathrm{~g} \mathrm{St}, 0.8 \mathrm{~g} \mathrm{MMA}$, and $1.0 \mathrm{~g}$ AA was added to the reactor. The mixture was stirred steadily at $200 \mathrm{rpm}$ and left at $70^{\circ} \mathrm{C}$ for $12 \mathrm{~h}$, before it was cooled to room temperature. The average particle size of the copolymer particles is $240 \pm 6 \mathrm{~nm}$, as determined from transmission electron microscopy (TEM, JEM-1200EX II, Japan). The composition of the copolymer was obtained by ${ }^{1} \mathrm{H}-\mathrm{NMR}$ spectral. A sample of the latex particles was centrifuged at $4000 \mathrm{rpm}$ for $40 \mathrm{~min}$ after the polymerization was completed and then washed with distilled water. After repetition of this, the particles were dried $48 \mathrm{~h}$ under vacuum at $60^{\circ} \mathrm{C}$. The ${ }^{1} \mathrm{H}-\mathrm{NMR}$ spectra of the copolymer sample were recorded with a $500 \mathrm{MHz}$ high-resolution NMR spectrometer (AVANCE 500) using deuterated chloroform as solvent. ${ }^{1} \mathrm{H}-\mathrm{NMR}$ spectra data showed that the copolymer obtained here consisted of 92, 3.85, and $4.15 \mathrm{wt} \%$ of St, MMA, and AA unit, respectively.

2.4. Preparation and Characterization of Surfactant-Free HIPEs. Appropriate VER-St, VER-MMA, or mixture of St and DVB as organic phase was added to a $100 \mathrm{~mL}$ beaker and was stirred with a magnetic stirrer at $400 \mathrm{rpm}$ for $5 \mathrm{~min}$ at $25^{\circ} \mathrm{C}$. Aqueous phase containing $\mathrm{P}$ (St-co-MMA-co-AA) particles of $1.0 \mathrm{wt} \%$ and $\mathrm{NaCl}$ of $0.2 \mathrm{~mol} \mathrm{~L}^{-1}$ was gradually added to the organic phase. The total liquid volume of each emulsion was kept at $30 \mathrm{~mL}$. The mixture was further stirred for $1 \mathrm{~min}$ after the addition of aqueous phase was completed. The component of the runs was shown in Tables 1 and 2. The resulting emulsions were named as SVX-Y, MVX-Y, or SDXY. SV (MV or SD) means VER-St (VER-MMA or the mixture 
TABle 1: Poly-Pickering-HIPEs via varied VEO concentrations in VER-St ${ }^{\mathrm{a}}$

\begin{tabular}{lccc}
\hline Run & $\mathrm{VEO}^{\mathrm{b}} / \mathrm{wt} \%$ & $\mathrm{Dv}^{\mathrm{c}} / \mu \mathrm{m}$ & $E^{\mathrm{d}} / \mathrm{MPa}$ \\
\hline SV0-90 & 0 & 90 & 2.1 \\
SV10-90 & 10 & 68 & 12.2 \\
SV20-90 & 20 & 40 & 16.8 \\
SV40-90 & 40 & 18 & 24.6 \\
SV50-90 & 50 & - & -
\end{tabular}

${ }^{\mathrm{a}} \mathrm{The} \mathrm{NaCl}$ concentration (relative to the aqueous phase) was $0.2 \mathrm{~mol} \mathrm{~L}^{-1}$, the copolymer particles content (relative to the aqueous phase) was $1.0 \mathrm{wt} \%$, and the aqueous phase volume fraction was $90 \%$.

${ }^{\mathrm{b}}$ Relative to the organic phase.

${ }^{\mathrm{c}}$ Average void diameter, calculated from SEM images.

delastic modulus.

TABLE 2: With VER-St or VER-MMA as organic phase, polyPickering-HIPEs were prepared via varied aqueous phase volume fraction ${ }^{\mathrm{a}}$.

\begin{tabular}{lccc}
\hline Run $^{\mathrm{b}}$ & $f_{w}{ }^{\mathrm{c}} / \%$ & $\mathrm{Dv}^{\mathrm{d}} / \mu \mathrm{m}$ & $E^{\mathrm{e}} / \mathrm{MPa}$ \\
\hline SV20-80 & 80 & 17 & 24.8 \\
SV20-90 & 90 & 40 & 16.8 \\
SV20-95 & 95 & 63 & 1.1 \\
SV20-97 & 97 & - & - \\
MV20-80 & 80 & 20 & 22.3 \\
MV20-90 & 90 & 41 & 15.4 \\
MV20-95 & 95 & 122 & 1.8 \\
MV20-97 & 97 & - & - \\
\hline
\end{tabular}

${ }^{\mathrm{a}}$ The $\mathrm{NaCl}$ concentration (relative to the aqueous phase) was $0.2 \mathrm{~mol} \mathrm{~L}^{-1}$, and the copolymer particles content (relative to the aqueous phase) was $1.0 \mathrm{wt} \%$.

bSV20-80, SV20-90, SV20-95, and SV20-97: VER-St containing VEO of $20 \mathrm{wt} \%$ as organic phase; MV20-80, MV20-90, MV20-95, and MV20-97: VER-MMA containing VEO of $20 \mathrm{wt} \%$ as organic phase.

cAqueous phase volume fraction.

${ }^{\mathrm{d}}$ Average void diameter, calculated from SEM images.

eElastic modulus.

of St and DVB) was used as the organic phase; $\mathrm{X}$ represents the concentration of the crosslinking agent (VEO or DVB) in the organic phase; $\mathrm{Y}$ represents the water phase volume percentage in the emulsion.

Stability of HIPEs was evaluated by measuring the backscattering of monochromatic light $(\lambda=880 \mathrm{~nm})$ from the suspension employing an optical analyzer, Turbiscan Lab Expert (Formulaction, France). As soon as the HIPEs were prepared, about $22 \mathrm{~mL}$ HIPE was transferred to a flat-bottomed cylindrical glass tube $(70 \mathrm{~mm}$ height, $27.5 \mathrm{~mm}$ external diameter), then the tube was placed in the instrument, and the backscattering of light from emulsion was periodically measured along the height at $25^{\circ} \mathrm{C}$. The results are presented as the sedimentation profile, that is, backscattering data versus time. The photographs of the samples were taken on digital camera (Coolpix-L1, Nikon).

2.5. Preparation and Characterization of Poly-PickeringHIPEs. Stable HIPEs were initiated by $1 \mathrm{wt} \%$ (relative to the aqueous phase) APS and polymerized in water bath at $70^{\circ} \mathrm{C}$

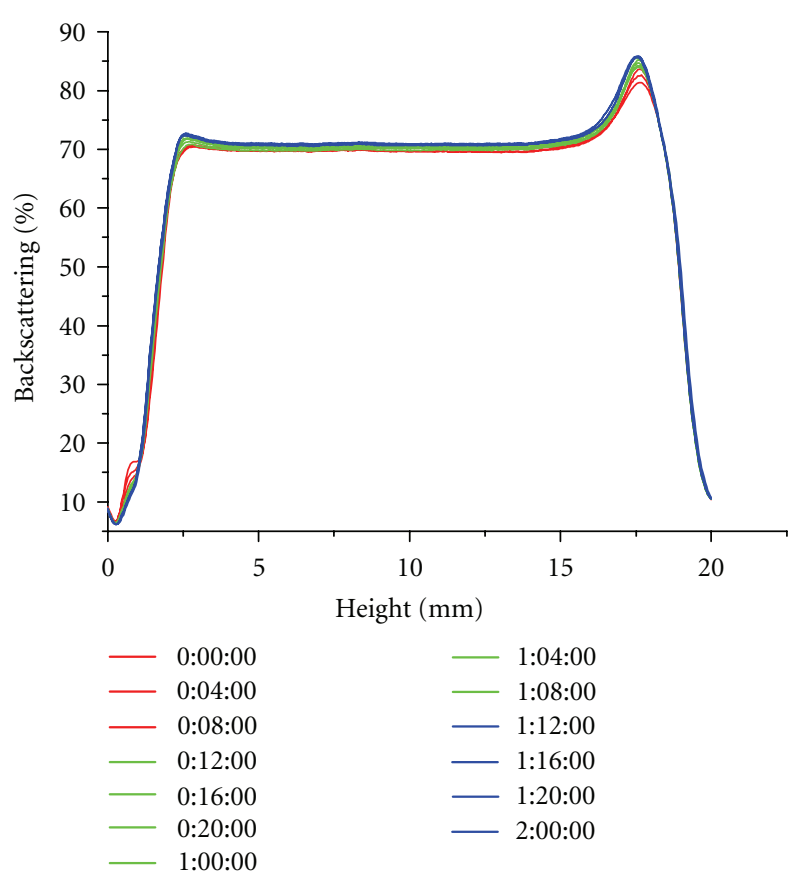

FIgURE 2: Backscattering of the HIPE stabilized by P(St-co-MMAco-AA) copolymer particles. VER-St was used as organic phase.

for $24 \mathrm{~h}$. The resulting poly-Pickering-HIPEs were removed from the molds and dried in vacuum at $70^{\circ} \mathrm{C}$ for $24 \mathrm{~h}$ then extracted in Soxhlet apparatus with distilled water to remove any impurities. Finally, the resulting polymers were dried to constant weight in vacuum at $70^{\circ} \mathrm{C}$.

The morphology of polyHIPEs was detected by a JSM-6360LV SEM. Samples were mounted on aluminum studs using adhesive graphite tape and sputter coated with approximately $5 \mathrm{~nm}$ of gold before analysis. The average void diameters of the poly-Pickering-HIPEs were performed using image analysis software Image J (NIH image) [26].

A Sans Universal Testing Machine equipped with a $20 \mathrm{kN}$ load cell (Shenzhen SANS Testing Machine Co. Ltd., Shenzhen, China) was used to measure mechanical properties in compression. The samples were loaded at a rate of $1 \mathrm{~mm} / \mathrm{min}$. Five samples of $20 \mathrm{~mm}$ in diameter and $10 \mathrm{~mm}$ in height were tested for each poly-Pickering-HIPE. The samples were loaded until a displacement of half the height of the examined sample was reached. The elastic modulus $(E)$ was determined from the initial linear slope of the stress/strain plot.

\section{Results and Discussion}

First of all, the emulsification of water and VER-St (containing $10 \mathrm{wt} \% \mathrm{VEO}$ ) was examined with $\mathrm{P}$ (St-co-MMAco-AA) particles as stabilizer. Milky-white W/O emulsion (HIPE SV10-90) was formed after stirring was ceased. The Turbiscan analysis of the emulsion showed that the backscattering data of the emulsion was stable, as shown in Figure 2. This means that with VER-St as organic phase, stable w/o HIPE can be formed. 

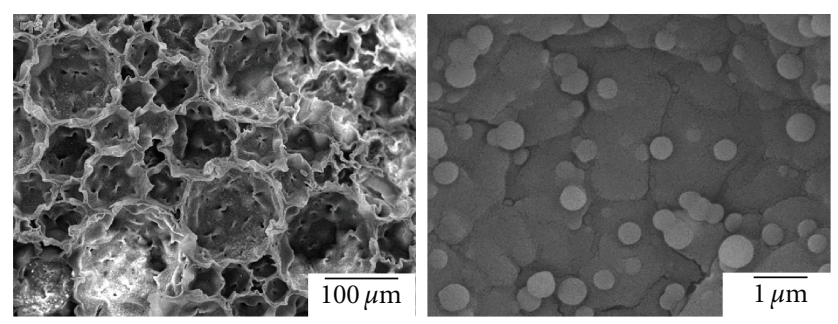

SV10-90
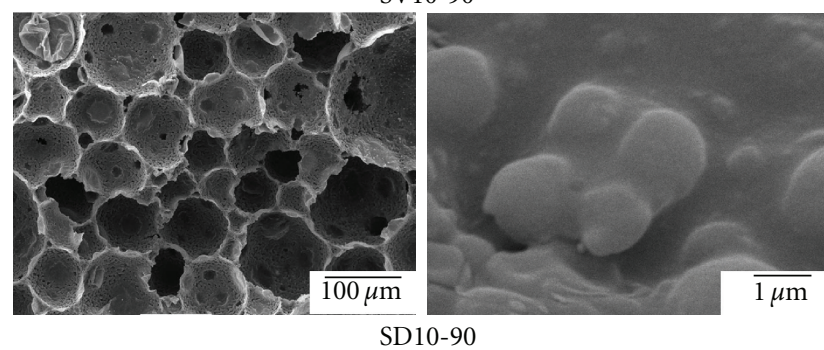

FIgure 3: SEM images of the poly-Pickering-HIPEs. The water volume fraction of the Pickering emulsion was 90 vol\%. SV10-90: VEO as crosslinker, $10 \mathrm{wt} \%$ based on the organic phase, (SD10-90): DVB as crosslinker, $10 \mathrm{wt} \%$ based on the organic phase.

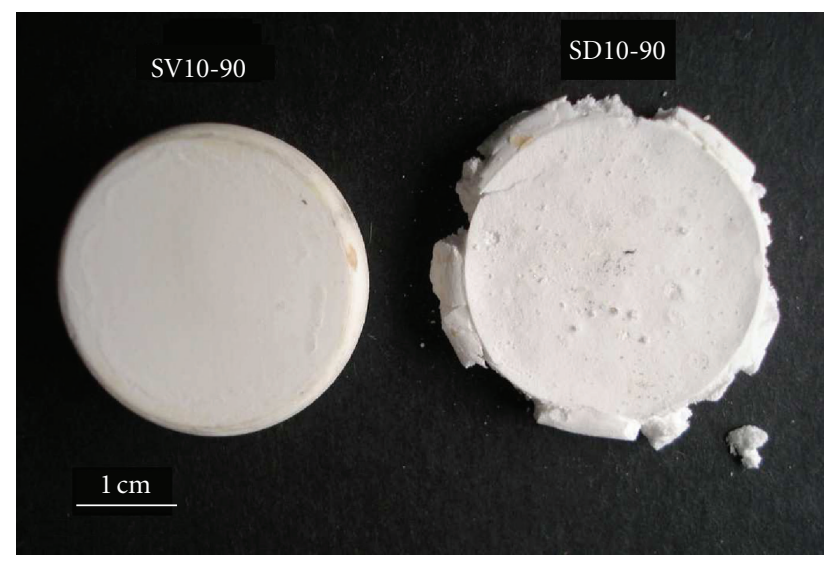

Figure 4: Photograph of the poly-Pickering-HIPEs which were compressed $50 \%$.

Initiating the HIPEs SV10-90 and SD10-90, polyPickering-HIPEs SV10-90 and SD10-90 were obtained, respectively, as shown in Figure 3. The average void diameter (Dv) of the poly-Pickering-HIPEs SV10-90 was $68 \mu \mathrm{m}$, and this value was similar to that $(64 \mu \mathrm{m})$ of the polyPickering-HIPEs SD10-90. However, the E (12.2 MPa) of poly-Pickering-HIPE SV10-90 was much higher than that (6.4 MPa) of the poly-Pickering-HIPE SD10-90 and was also much higher than the $E(3.0 \mathrm{MPa})$ of the PS-based polyHIPE prepared with the HIPE having internal phase volume fraction of $89 \%$ [27]. Moreover, when the samples were loaded until a displacement of half the height of the examined sample was reached, poly-Pickering-HIPE SD10-90 was crushed, while no crack was observed in poly-Pickering-HIPE SV10-90, as shown in Figure 4. This means that both $E$ and toughness of poly-Pickering-HIPEs
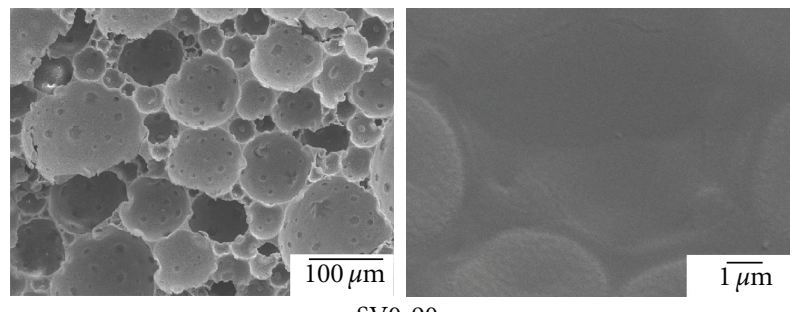

SV0-90
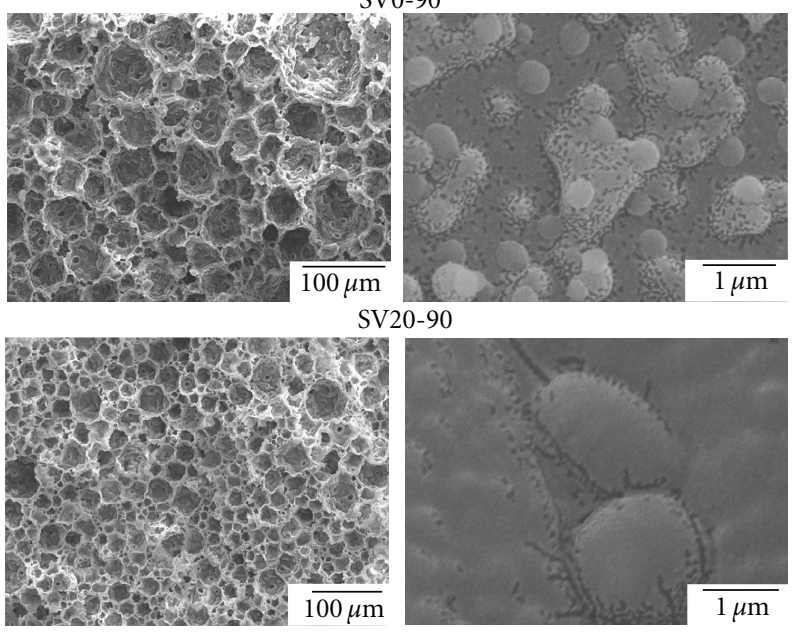

SV40-90

FIGURE 5: SEM images of VER-based poly-Pickering-HIPEs which were obtained with varied VEO concentrations.

can be enhanced appreciably with VEO replacing DVB as crosslinker.

The effect of VEO on the morphology and $E$ of VER-Stbased poly-Pickering-HIPEs was investigated. As shown in Table 1, poly-Pickering-HIPEs with organic phase containing VEO of $0,10,20$, and $40 \mathrm{wt} \%$, respectively, were prepared (see Figures 3 and 5). It was found that increasing VEO from 0 to $40 \mathrm{wt} \%$ resulted in an increase in $E$ by about $22 \mathrm{MPa}$ (Figure 6) and a decrease in Dv by about $70 \mu \mathrm{m}$. The decrease in Dv was attributed to the fact that increasing VEO concentration in the organic phase caused an increase in the viscosity of the organic phase, which enhanced the emulsion stability $[28,29]$ and therefore led to an increasing number of small droplets and the narrow distribution of droplet size. These resulted in the decrease in poly-Pickering-HIPE void diameter and its narrow distribution (see Figure 7), since the porous polymer was effectively a replica of the emulsion structure by gel formation. However, the poly-PickeringHIPE with organic phase containing $50 \mathrm{wt} \%$ of VEO could not be prepared, because the viscosity of the organic phase was too high so that the aqueous phase could not be wrapped effectively by the organic phase.

Moreover, increasing the VEO concentration in organic phase also affected the morphology of the void surface. When VER-St containing 0 or $10 \mathrm{wt} \%$ VEO was used as organic phase, there were no pores in the void surface. However, when the VER-St containing VEO of 20 or $40 \mathrm{wt} \%$ was used, in the void surface many pores with diameters ranged from 20 to $100 \mathrm{~nm}$ were observed (see Figure 4). In all likelihood, 


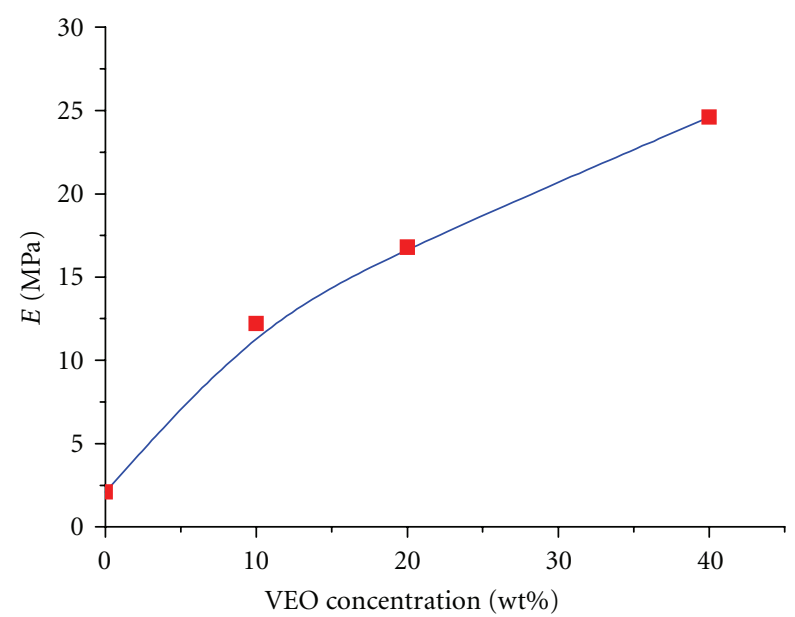

FIGURE 6: Effect of VEO concentration in the organic phase on the elastic modulus of the poly-Pickering-HIPEs. The water volume fraction of the emulsion was $90 \mathrm{vol} \%$, and the $\mathrm{NaCl}$ and copolymer particle concentration relative to aqueous phase were $0.2 \mathrm{~mol} \mathrm{~L}^{-1}$ and $1.0 \mathrm{wt} \%$, respectively.

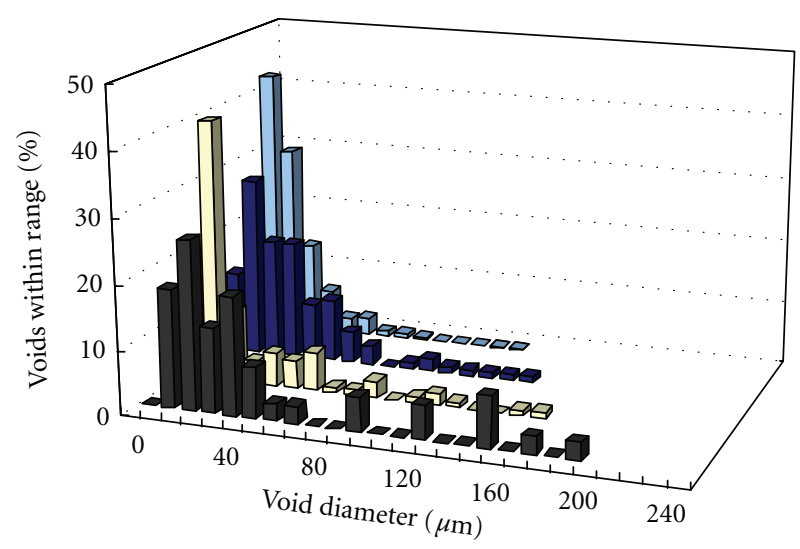

FIgURE 7: Void diameter distributions of poly-Pickering-HIPEs obtained with varied VEO concentrations. From front to back: SV090, SV10-90, SV20-90, and SV40-90.

the cause of the pore formation was the volume contraction of VER-St during the polymerization, and the extent of shrinkage highly depended on VEO concentration [30,31].

Using VER-St (or VER-MMA) containing VEO of $20 \mathrm{wt} \%$ as organic phase, stable Pickering HIPEs with aqueous phase volume fraction of 80,90 , and 95 vol\% were obtained, respectively, as listed in Table 2. However, the Pickering HIPEs SV20-97 and MV20-97 having aqueous phase volume fraction of $97 \mathrm{vol} \%$ could not be prepared, because the aqueous phase was too much to be wrapped effectively by the organic phase. This phenomenon showed that the up limits of the aqueous phase volume fraction in these Pickering HIPEs were between 95 and 97 vol\% which was higher than that ( $\leq 92 \mathrm{vol} \%)$ of those Pickering HIPEs with inorganic particles (e.g., $\mathrm{SiO}_{2}$ or $\mathrm{TiO}_{2}$ ) as stabilizer $[32,33]$. The polymerizing stable Pickering HIPEs, polyPickering-HIPEs SV20-80-SV20-95 and MV20-80-MV20-95

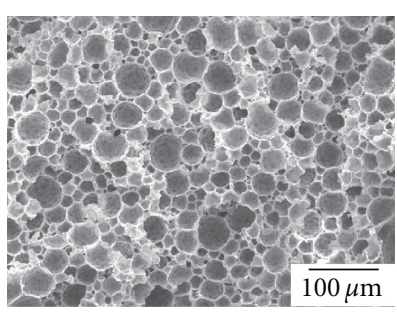

SV20-80

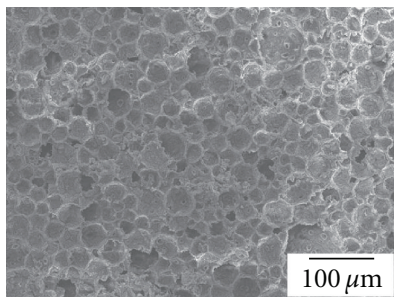

MV20-80

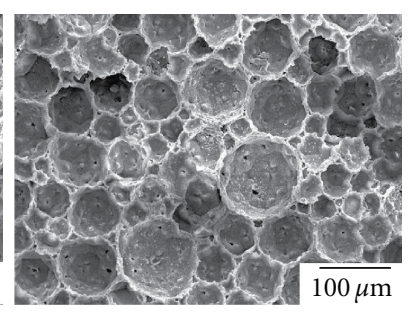

SV20-95

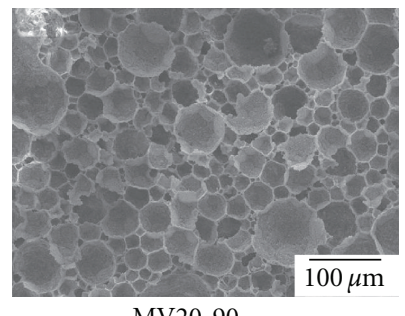

MV20-90

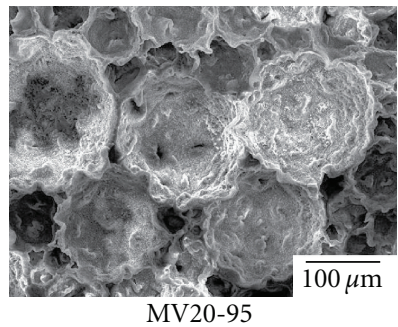

FIgure 8: SEM images for VER-based poly-Pickering-HIPEs obtained with different aqueous phase volume fraction.

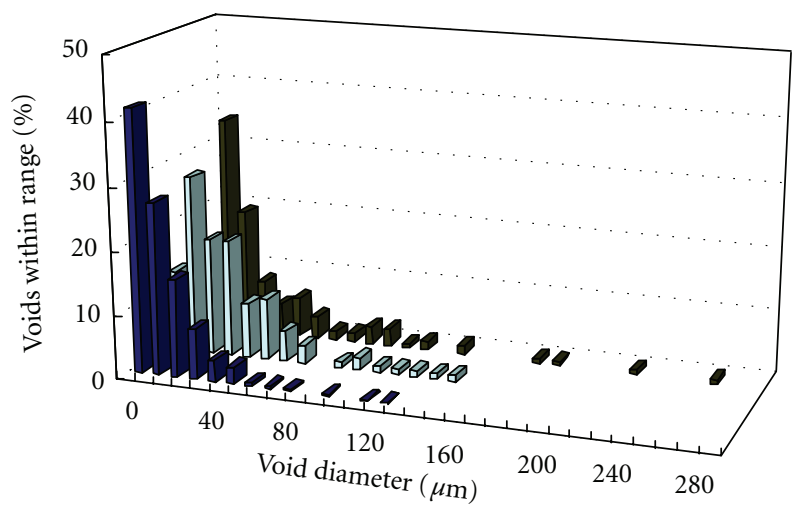

FIGURE 9: Void diameter distributions of poly-Pickering-HIPEs obtained with VER-St as organic phase and varied aqueous phase volume fraction. From front to back: SV20-80, SV20-90, and SV2095.

were obtained (as shown in Figures 3 and 8). It was found that increasing aqueous phase volume fraction resulted in an increase in Dv (see the detailed data in Table 2) and a broader distribution of Dv (see Figure 9). This result is consistent with the previous report about poly-Pickering-HIPEs with MMA and St as organic phase [3]. Compress analysis showed that increasing aqueous phase volume fraction caused a significant decrease in E (see Table 2 and Figure 10). This phenomenon was consistent with other researchers' 

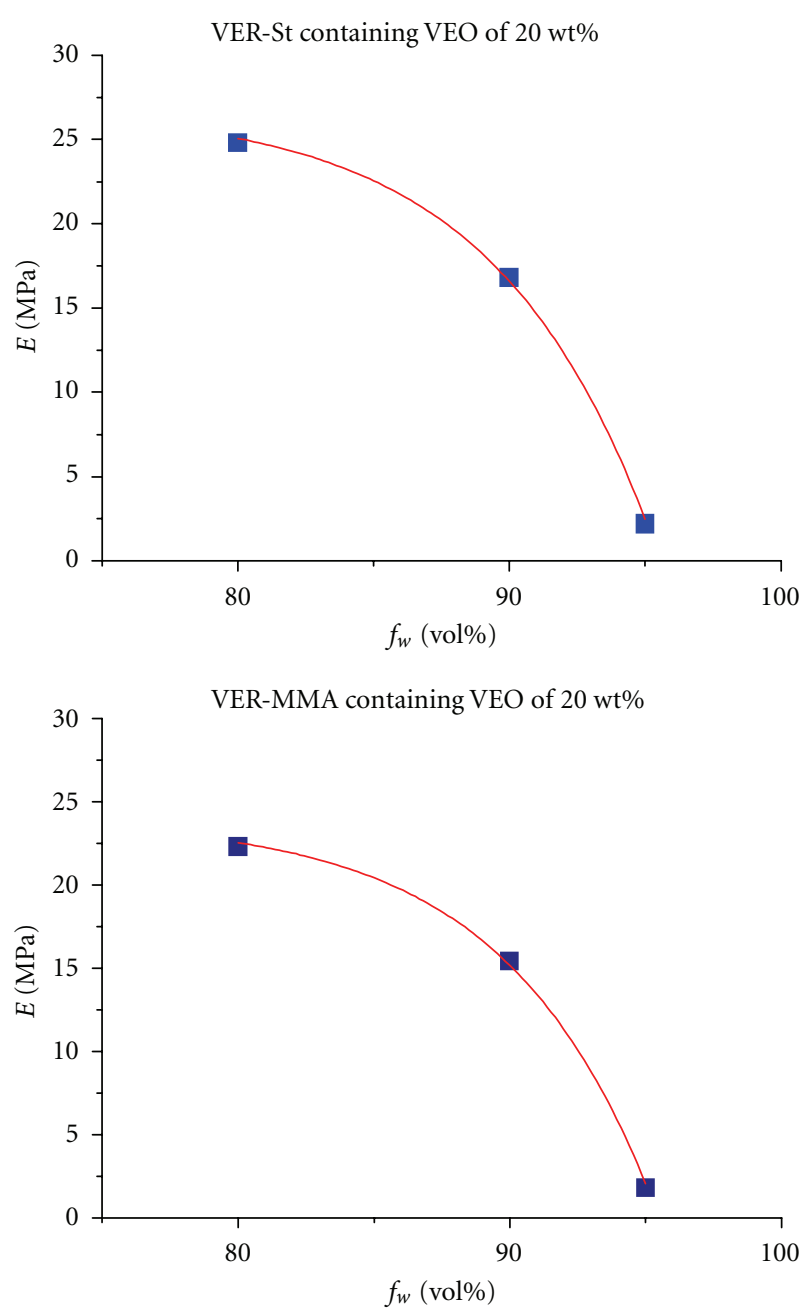

FIgURE 10: Effect of aqueous phase volume fraction on the elastic modulus of the poly-Pickering-HIPEs.

report [27] and could be due to the fact that increasing aqueous phase of the Pickering HIPEs resulted in a decrease in the density of the resulting porous polymers.

\section{Conclusions}

In this study, using VER as organic phase, poly-PickeringHIPEs having internal phase volume fraction up to $95 \mathrm{vol} \%$ were prepared. Compared to the poly-Pickering-HIPEs with DVB as crosslinker, VEO as crosslinker is conducive to the enhancement of both $E$ and toughness of the poly-PickeringHIPEs. The elastic modulus of these poly-Pickering-HIPEs was increased by increasing the VEO concentration in the organic phase, while decreased by increasing aqueous phase volume fraction. Increasing VEO concentration in the organic phase resulted in a decrease in the average void diameter and a narrow void diameter distribution of the resulting poly-Pickering-HIPEs. In addition, there were many small pores in the voids surface caused by volume contraction of VER during the polymerization, which highlights an opportunity for poly-Pickering-HIPEs to possess hierarchical structures without any additive agent.

\section{Acknowledgments}

This research was supported by the National Natural Scientific Foundation of China (51103042), the Fundamental Research Funds for the Central Universities, Innovation Program of Shanghai Municipal Education Commission (12ZZ056), Shanghai Leading Academic Discipline Project (Project no. B502), and Shanghai Key Laboratory Project (08DZ2230500).

\section{References}

[1] E. Ruckenstein, "Concentrated emulsion polymerization," Advances in Polymer Science, vol. 127, pp. 1-58, 1997.

[2] N. R. Cameron, "High internal phase emulsion templating as a route to well-defined porous polymers," Polymer, vol. 46, no. 5, pp. 1439-1449, 2005.

[3] S. Zhang and J. Chen, "PMMA based foams made via surfactant-free high internal phase emulsion templates," Chemical Communications, no. 16, pp. 2217-2219, 2009.

[4] S. Kovačič, K. Jeřábek, P. Krajnc, and C. Slugovc, "Ring opening metathesis polymerisation of emulsion templated dicyclopentadiene giving open porous materials with excellent mechanical properties," Polymer Chemistry, vol. 3, pp. 325$328,2012$.

[5] S. Kovačič, P. Krajnc, and C. Slugovc, "Inherently reactive polyHIPE material from dicyclopentadiene," Chemical Communications, vol. 46, no. 40, pp. 7504-7506, 2010.

[6] Y. Zhu, S. Zhang, Y. Hua, J. Chen, and C. P. Hu, "Hydrophilic porous polymers based on high internal phase emulsions solely stabilized by poly(urethane urea) nanoparticles," Polymer, vol. 51, no. 16, pp. 3612-3617, 2010.

[7] Y. Zhu, S. Zhang, J. Chen, and C. P. Hu, "High internal phase emulsions prepared with poly(urethane urea) aqueous nanodispersion at different temperatures," Journal of Polymer Science, Part A, vol. 48, no. 19, pp. 4356-4360, 2010.

[8] T. T. Li, H. R. Liu, L. Zeng et al., "Macroporous magnetic poly(styrene-divinylbenzene) nanocomposites prepared via magnetite nanoparticles-stabilized high internal phase emulsions," Journal of Materials Chemistry, vol. 21, pp. 1286512872, 2011.

[9] S. Wang, Z. Zhang, H. Liu, W. Zhang, Z. Qian, and B. Wang, "One-step synthesis of manganese dioxide/polystyrene nanocomposite foams via high internal phase emulsion and study of their catalytic activity," Colloid and Polymer Science, vol. 288, no. 9, pp. 1031-1039, 2010.

[10] J. Yang, G. Yang, H. Liu, L. Bai, and Q. Zhang, "Preparation and characterization of porous poly(vinyl ester) resin monoliths as separation media," Journal of Applied Polymer Science, vol. 119, no. 1, pp. 412-418, 2011.

[11] C. Canal, F. Gaboriau, A. Vílchez, P. Erra, M. J. Garcia-Celma, and J. Esquena, "Topographical and wettability effects of postdischarge plasma treatments on macroporous polystyrenedivinylbenzene solid foams," Plasma Processes and Polymers, vol. 6, no. 10, pp. 686-692, 2009.

[12] R. Molina, A. Vilchez, C. Canal, and J. Esquena, "Wetting properties of polystyrene/divinylbenzene crosslinked porous polymers obtained using W/O highly concentrated emulsions 
as templates," Surface and Interface Analysis, vol. 41, no. 5, pp. 371-377, 2009.

[13] S. Zhang and J. Chen, "Synthesis of open porous emulsiontemplated monoliths using cetyltrimethylammonium bromide," Polymer, vol. 48, no. 11, pp. 3021-3025, 2007.

[14] S. Zhang, J. Chen, and V. T. Perchyonok, "Stability of high internal phase emulsions with sole cationic surfactant and its tailoring morphology of porous polymers based on the emulsions," Polymer, vol. 50, no. 7, pp. 1723-1731, 2009.

[15] H. Zhang and A. I. Cooper, "Synthesis and applications of emulsion-templated porous materials," Soft Matter, vol. 1, no. 2, pp. 107-113, 2005.

[16] S. M. Zhang, Y. Zhu, Y. Hua, C. Jegat, J. D. Chen, and T. Mohamed, "Stability of surfactant-free high internal phase emulsions and its tailoring morphology of porous polymers based on the emulsions," Polymer, vol. 52, pp. 4881-4890, 2011.

[17] S. D. Kimmins and N. R. Cameron, "Functional porous polymers by emulsion templating: recent advances," Advanced Functional Materials, vol. 21, no. 2, pp. 211-225, 2011.

[18] S. Zhang, J. Chen, and V. T. Perchyonok, "PolyHIPEs as novel media for conventional free radical chemistry," Letters in Organic Chemistry, vol. 5, no. 4, pp. 304-307, 2008.

[19] S. Zhang, J. Chen, I. N. Lykakis, and V. T. Perchyonok, "Streamlining organic free radical synthesis through modern molecular technology: from polymer supported synthesis to microreactors and beyond," Current Organic Synthesis, vol. 7, no. 2, pp. 177-188, 2010.

[20] S. Zhang, J. Chen, and V. T. Perchyonok, "Highly facile regioselective ring opening of epoxides to halohydrins in the presence of polyhipes (CTAB) and $\beta$-cyclodextrins (TSAX) in water," Current Organic Chemistry, vol. 15, no. 15, pp. 2690 2693, 2011.

[21] G. Y. Wang, Y. L. Wang, and C. P. Hu, "Interpenetrating polymer networks of polyurethane and graft vinyl ester resin: polyurethane formed with toluene diisocyanate," European Polymer Journal, vol. 36, no. 4, pp. 735-742, 2000.

[22] L. H. Fan, C. P. Hu, and S. K. Ying, "Thermal analysis during the formation of polyurethane and vinyl ester resin interpenetrating polymer networks," Polymer, vol. 37, no. 6, pp. 975-981, 1996.

[23] L. H. Fan, C. P. Hu, Z. P. Zhang, and S. K. Ying, "Polymerization kinetics of polyurethane and vinyl ester resin interpenetrating polymer networks by using fourier transform infrared spectroscopy," Journal of Applied Polymer Science, vol. 59, no. 9, pp. 1417-1426, 1996.

[24] G. Y. Wang, M. Q. Zhu, and C. P. Hu, "Interpenetrating polymer networks of polyurethane and graft vinyl ester resinpolyurethane formed with diphenylmethane diisocyanate," Journal of Polymer Science, vol. 38, pp. 136-144, 2000.

[25] S. Zhang, J. Chen, and M. Taha, "Synthesis of monodisperse styrene/methyl methacrylate/ acrylic acid latex using surfactant-free emulsion copolymerization in air," Journal of Applied Polymer Science, vol. 114, no. 3, pp. 1598-1605, 2009.

[26] R. J. Carnachan, M. Bokhari, S. A. Przyborski, and N. R. Cameron, "Tailoring the morphology of emulsion-templated porous polymers," Soft Matter, vol. 2, no. 7, pp. 608-616, 2006.

[27] A. Menner, R. Powell, and A. Bismarck, "Open porous polymer foams via inverse emulsion polymerization: should the definition of high internal phase (ratio) emulsions be extended?" Macromolecules, vol. 39, no. 6, pp. 2034-2035, 2006.
[28] K. J. Lissant, Ed., Emulsions and Emulsion Technology Part 1, Marcel Dekker, New York, NY, USA, 1974.

[29] B. P. Binks and S. O. Lumsdon, "Stability of oil-in-water emulsions stabilised by silica particles," Physical Chemistry Chemical Physics, vol. 1, no. 12, pp. 3007-3016, 1999.

[30] Y. J. Huang and C. M. Liang, "Volume shrinkage characteristics in the cure of low-shrink unsaturated polyester resins," Polymer, vol. 37, no. 3, pp. 401-412, 1996.

[31] W. Li and L. J. Lee, "Shrinkage control of low-profile unsaturated polyester resins cured at low temperature," Polymer, vol. 39, no. 23, pp. 5677-5687, 1998.

[32] V. O. Ikem, A. Menner, and A. Bismarck, "High internal phase emulsions stabilized solely by functionalized silica particles," Angewandte Chemie-International Edition, vol. 47, no. 43, pp. 8277-8279, 2008.

[33] A. Menner, V. Ikem, M. Salgueiro, M. S. P. Shaffer, and A. Bismarck, "High internal phase emulsion templates solely stabilised by functionalised titania nanoparticles," Chemical Communications, no. 41, pp. 4274-4276, 2007. 

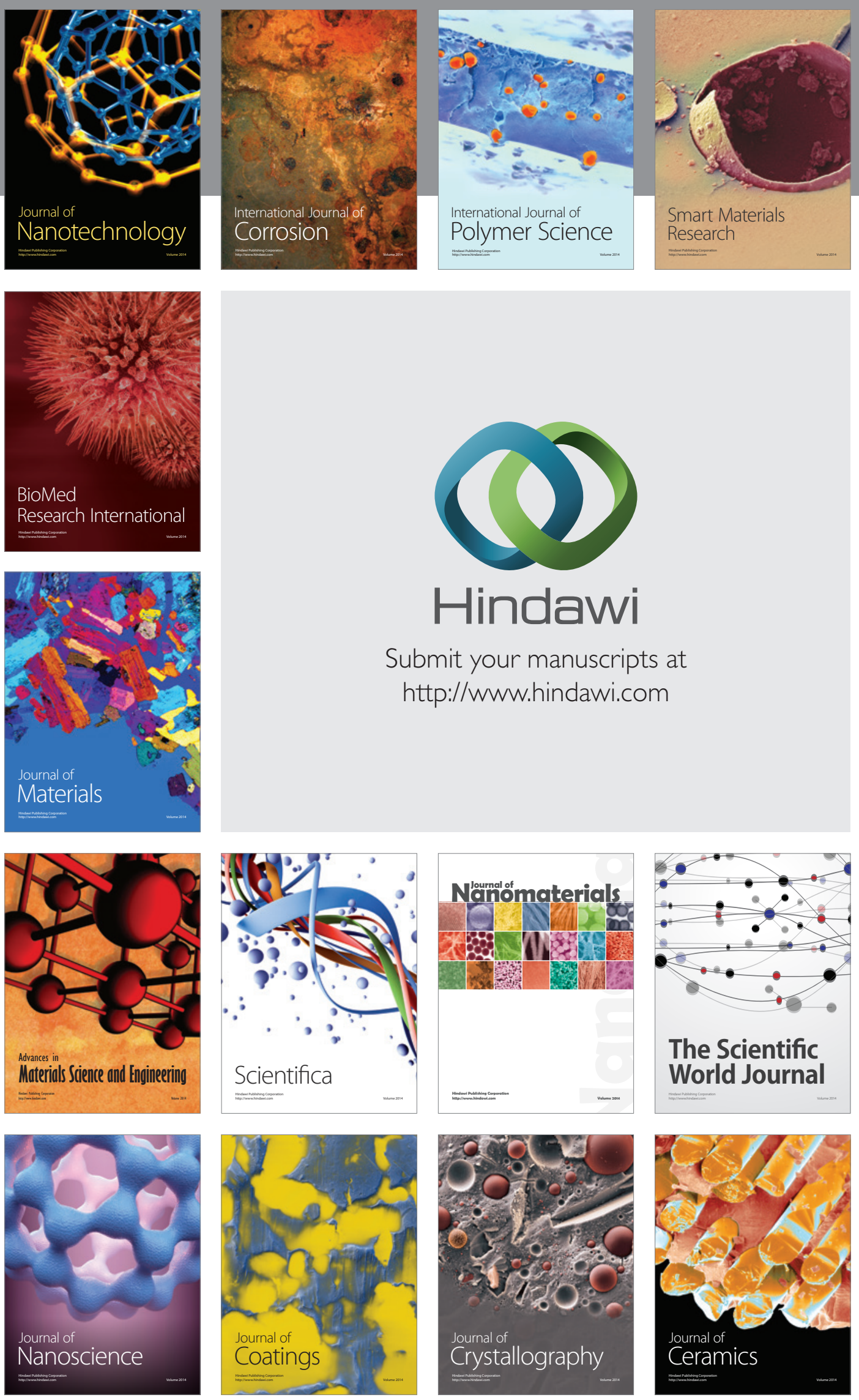

The Scientific World Journal

Submit your manuscripts at

http://www.hindawi.com

\section{World Journal}

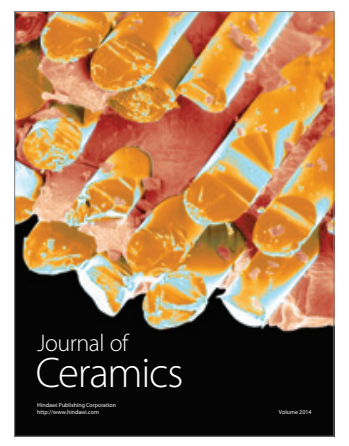

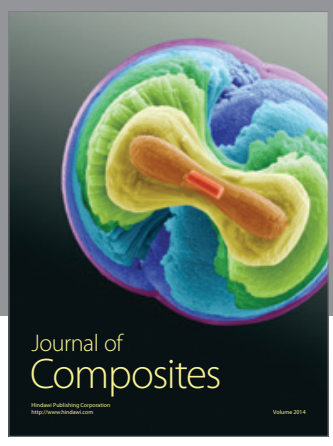
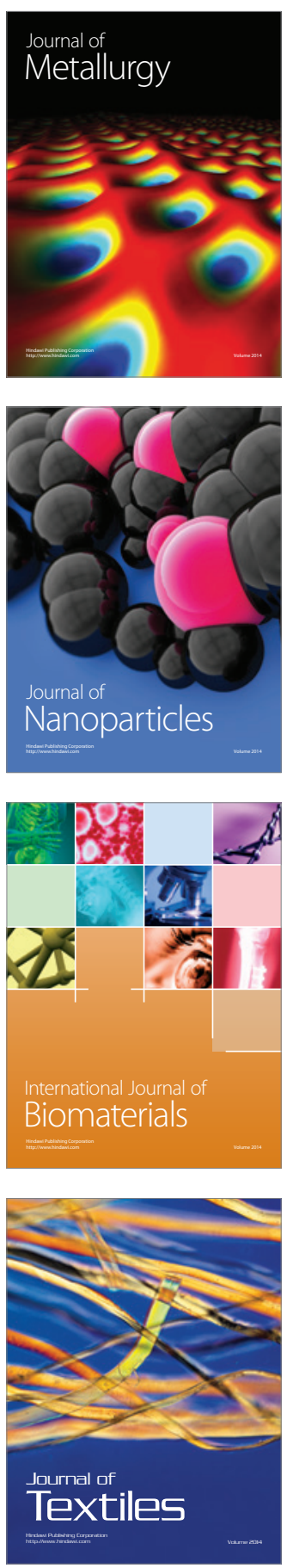